Open Access

\title{
The relationship between the parenteral dose of fish oil supplementation and the variation of liver function tests in hospitalized adult patients \\ (1) ${ }_{\text {crosenata }}$
}

Maria B. Badia-Tahull ${ }^{1 *}$, Elisabet Leiva-Badosa' ${ }^{1}$, Ramon Jodar-Masanes ${ }^{1}$, Josep Maria Ramon-Torrell ${ }^{2}$ and Josep Llop-Talaveron'

\begin{abstract}
Background: Hepatic dysfunction is a complication associated with parenteral nutrition (PN). Our primary objective was to study the relationship between doses of intravenous fish oil (FO) emulsion in PN and the variation in the main liver function tests (LFTs) in hospitalized PN-treated adults. As a secondary objective, we studied the safety of FO administration.

Methods: We conducted a retrospective study in adult patients receiving FO supplementation in PN. Demographic, nutritional and safety variables were collected. Variation of LFTs was defined as the difference between values just before the first administration of FO and values at the end of PN. A multiple linear regression was performed to study the association between PN-lipids (FO or vegetable) and the variation of each LFT; the following variables were used to adjust the effect of lipids: sepsis, length of stay in the intensive care unit and lipids dose. Student t-test was used to study safety variables. Data were analyzed using SPSS 19.0.

Results: Patients (53, median age 68 years (24-90); $62 \%$ men) with the principal diagnosis of digestive neoplasm $(42 \%)$ received PN for a median of 19 (7-75) days. In the multivariate analysis, the amount of FO was related to a decrease in gamma-glutamyl transferase (GGT) ( $\mathrm{B}=-2.23 ; \mathrm{Cl} 95 \%=-4.41 /-0.05)$, a decrease in alkaline phosphatase (AP) $(B=-1.23 ; C \mid 95 \%=-2.07 /-0.37)$, and a decrease in alanine aminotransferase (ALT) $(B=-0.82 ; C 195 \%=-1.19 /-0.44)$. No differences were found in safety variables.
\end{abstract}

Conclusions: GGT, AP and ALT improved with FO PN-supplementation. Moreover, the improvement was greater when the doses of FO were higher. FO administration in PN is safe.

Keywords: Liver function tests, Fish oil emulsion, Fatty acids, Omega-3 fatty acids, Parenteral nutrition

\section{Background}

Fat is increasingly recognized as a central feature of many biological processes [1]. Lipid emulsions are an essential part of parenteral nutrition (PN), both as an energy supply and as a source of essential fatty acids (FAs) [2]. Furthermore, they are involved in the structure and function of cell membranes and receptors, modifying gene expression and modulating the inflammatory and immune responses [2]. Moreover, FAs are precursors of

\footnotetext{
* Correspondence: mbadia@bellvitgehospital.cat

${ }^{1}$ Pharmacy Department, Hospital Universitari Bellvitge. IDIBELL. C/Feixa Llarga s/n. 08907 L'Hospitalet de Llobregat, Barcelona, Spain

Full list of author information is available at the end of the article
}

prostaglandins and other eicosanoids and have therefore important metabolic functions [2].

The development of significant hepatic dysfunction is a well-recognized complication associated with PN administration [2, 3]. A spectrum of hepatobiliary disorders, ranging from simple steatosis to cholestasis, cholelithiasis, hepatic fibrosis and subsequent progression to cirrhosis, portal hypertension and end-stage liver disease, can occur [4]. This disorder is characterized by elevated serum levels of total bilirubin (BIL), alkaline phosphatase (AP), gammaglutamyl transferase (GGT) and transaminases (alanine aminotransferase [ALT] and/or aspartate aminotransferase [AST]) $[2,3,5]$. Cholestasis and hepatocellular damage are well-known complications caused by long-term $\mathrm{PN}$, 
especially in the neonatal and pediatric population but also in adult patients with short bowel syndrome (SBS). In adult patients, this hepatic dysfunction may be present as cholestasis, hepatosteatosis or cholelithiasis [5]. Parenteral nutrition-associated liver disease (PNALD) is well established in patients with long-term home PN treatment. But it is also true that those patients who only receive PN during hospitalization have the potential to develop alterations in their LFTs [6, 7]. Although liver failure develops slowly over months or years, steatosis and steatohepatitis may develop within weeks in acutely ill patients [4]. This liver alteration is normally transient, and may not progress to a worse condition. However, it could add to those liver alterations caused by drugs and specific clinical situations and might also pose a limitation to a correct intravenous nutrient supply.

The mechanisms leading to PNALD remain largely unknown but are likely to be multifactorial $[2,5,8]$. Established risk factors for its development are duration of PN and number of septic episodes [9], but several aspects of the parenteral formula have also been thought to be responsible for these liver test abnormalities [10, 11]. Macronutrient excess, i.e., feeding beyond the liver's ability to use carbohydrate and fat, can cause accumulation of by-products in the liver and result in hepatic injury. Another cause is the source of fat used. Lipids coming from soybean oil (SO) have high levels of $\omega-6$ and are associated with liver toxicity, mainly with long-term utilization. After them, median chain triglycerides (MCT) were combined with $\mathrm{SO}$ in order to provide energy quickly and avoid some of the secondary effects detected with SO. Olive oilbased lipids were introduced later with potentially fewer lipoperoxidation problems; and recently, lipids derived from fish have been reported to have anti-inflammatory activity. Combinations of all these lipids have appeared on the market and their use has been extended. The longterm use of these emulsions has permitted their correlation with different types of PNALD and with recovery from PNALD in premature and home parenteral nutrition patients. Initially, in our hospital, only SO was used but, as soon as different products for inflammatory conditions were marketed, we also introduced them in our clinical protocol. These decisions were based on clinical experience, coming mainly from the clinical guidelines of the European Society of Parenteral and Enteral Nutrition (ESPEN). In addition, vegetable oil-based lipid emulsions contain significant quantities of phytosterols that accumulate in patients' serum and result in cholelithiasis [10]. Long-term use of soybean oil (SO)-based emulsions leads to a progressive increase of phytosterol content in cell membranes and plasma lipoproteins which has been associated with the onset of PNALD in children receiving long-term PN [9]. SO also depletes vitamin E from plasma lipoproteins and may contribute to hepatic injury [12].
Inflammation is another crucial factor and $\omega-6$ long chain polyunsaturated fatty acids (PUFAs) have been shown to worsen inflammatory states and to have immunosuppressive effects [9]. The peroxidative nature of $\omega-6$ could increase inflammatory activity in an infectious (sepsis) or non-infectious episode [4].

Some evidence has suggested that the administration of FO-based lipid emulsions may be useful in reversing PNALD [13]. These emulsions are primarily composed of $\omega-3$ PUFAs. In Europe, FO can be used both as part of a standard lipid emulsion or as a supplement, alone or in combination with other lipid emulsions. FO is mostly used to attenuate the patient's inflammatory state and not to modify liver function. The purpose of this study was to test, in routine clinical practice, whether there is an influence of FO on LFTs in adult patients treated with PN during hospitalization.

Our main objective was therefore to study the relationship between the doses of intravenous FO emulsion supplementation and the variation in the main LFTs in adult patients receiving PN during hospitalization. As a secondary objective, we studied the safety of the doses of FO given.

\section{Methods}

This was a retrospective study conducted over a 16month period in a 600-bed, third-level teaching hospital. Patients included in the study met the following criteria: older than 17 years of age, receiving FO supplementation in $\mathrm{PN}$ and not receiving enteral nutrition or oral food. Those patients with liver disease previous to PN initiation were excluded.

Data were collected in several categories: demographic variables (gender, age, height and weight); clinical variables (diagnosis, indication for PN administration, sepsis episodes, length of stay [LOS] in intensive care unit [ICU] and death); and nutritional variables (length of treatment with PN and amount of parenteral macronutrients administered). Nutritional data were collected from the database program of PN in the Pharmacy Department. Analytical and clinical data were collected from the hospital's computerized database.

Lipids were given as: Clinoleic ${ }^{\circ}$ (Baxter, Lessines, Belgium) based on a mixture of purified olive oil (80 \%) and SO (20 \%); SMOFlipid ${ }^{\circ}$ (Fresenius Kabi, Bad Homburg, Germany) based on SO (30\%), MCT (30\%), olive oil $(25 \%)$ and FO (15\%); and Omegaven (Fresenius Kabi, Bad Homburg, Germany), an emulsion exclusively of FO. In our study the amount of lipids administered by source, either vegetable (SO, MCT, olive oil) or not (FO), was studied separately.

In our routine practice, according to our protocol, lipids in PN are administered as a maximum dose of $1 \mathrm{~g} / \mathrm{kg} /$ day, although this dose is reduced in situations of 
hypertriglyceridemia. Primarily, when the patient presents an inflammatory state, SMOFlipid ${ }^{\circ}$ is used. However, in those patients with a high inflammatory state a combination of Omegaven ${ }^{\circ}$ and Clinoleic ${ }^{\circ}$ is used in order to achieve higher proportions of FO (with a maximum of $50 \%$ of the dose). To classify the inflammatory state, we used C-reactive protein (CRP) $[\mathrm{mg} / \mathrm{L}]$ and prealbumin $[\mathrm{mg} / \mathrm{L}]$ as biochemical and immunology parameters, since they are acute phase proteins. Nevertheless, in the extreme situation, even when the prealbumin is not so low, we maintained high proportions of omega-3 if CRP values were high. A third parameter that we have taken into account is plasma triglycerides $[\mathrm{mmol} / \mathrm{L}]$ because of the better clearance of omega-3 lipids [4]. In addition to these parameters we have also taken into account the clinical situation of the patient. In cases of hypertriglyceridemia where the total dose is reduced, lipids are given only as Omegaven ${ }^{\circ}$. Once FO emulsion was introduced in the nutritional formula, it was continued until the end of PN treatment. So, the same patient could receive different types of lipids according to their clinical situation All patients studied received a fixed standard daily supplementation of trace elements and vitamins.

\section{Assessment}

Venous blood samples were taken at the beginning of treatment with PN containing FO and twice a week thereafter. Liver function assessment was carried out with measurements of GGT, AP, ALT and BIL. On the other hand, safety variables were only determined at the beginning of the study and at the end of PN treatment; these variables were serum triglyceride (TG) levels and markers of coagulopathy: platelets count (PLC) and the international normalized ratio (INR).

\section{Statistical analysis}

For the statistical analysis, the principal variable chosen was the variation of LFTs. It was defined as the difference between LFT values just before the first administration of FO in the PN and the final values at the end of PN administration (results of these differences could be positive or negative according to an elevation or a diminution in the parameter, respectively).

Using this principal variable, two different statistical models were constructed:

\section{1) Univariate model}

The association between the variation in the liver parameters and the total dose of FO administered during the study $(\mathrm{g} / \mathrm{kg}$ ) was evaluated by one-way analysis of variance (ANOVA). Five categories were defined according to the variation found in each liver parameter: the values of the variations obtained were classified as high increase (including patients above with an increase superior to the $80^{\text {th }}$ percentile), moderate increase (from the $60^{\text {th }}$ to the $80^{\text {th }}$ percentile), no modification (between the $40^{\text {th }}$ and $60^{\text {th }}$ percentile), moderate decrease $\left(20^{\text {th }}\right.$ and $40^{\text {th }}$ percentile) and high decrease (with a variation of less than the $20^{\text {th }}$ percentile).

2) Multivariate model

A multiple linear regression model was constructed to study the relationship between each LFT variation as a continuous variable and the amount of FO given adjusted in accordance with four important variables of liver co-morbidity:

- Presence of sepsis when each liver parameter presented the highest value. According to the American College of Chest Physician/Society of Critical Care Medicine (ACCP/SCCM) consensus conference, sepsis was established when the patient presented at least two of the following criteria: (a) body temperature $\geq 38{ }^{\circ} \mathrm{C}$ or $\leq 36{ }^{\circ} \mathrm{C}$, (b) tachycardia with heart rate $\geq 90$ beats $/ \mathrm{min}$, (c) tachypnea with $\mathrm{paCO}_{2} \leq 32 \mathrm{mmHg}$ or mechanical ventilation; (d) leukocytes $>12 \times 10^{9}$ cells/L [14].

- LOS in ICU considered as the number of days that the patient stayed in ICU.

- Dose of lipids. This variable contained two different elements: FO and vegetable-based lipids. In order to avoid the co-linearity effect between them, the amount of FO was introduced in the statistical model adjusted by residuals.

Additionally, in order to check the safety of FO administration, differences between the beginning and the end values for safety variables (TG, PLC and INR) were studied with $\mathrm{t}$-test for paired samples.

All data were analyzed using SPSS 19.0 (SPSS INC, Chicago IL, USA). Statistical significance was reported with a $95 \%$ confidence interval at the conventional $\mathrm{p}<0.05$ level (two-tailed).

Written informed consent was considered not necessary for the study, as it was a non-interventional study. Parenteral nutrition and nutritional and safety variables were prescribed and collected according to clinical practice. Patient data were anonymized for the purposes of this analysis. Confidential patient information was protected according to national normatives. This manuscript has been revised for publication by the Clinical Research Ethics Committee of our Hospital.

\section{Results}

During the total study period (January 2010 to April 2011), 53 patients received PN-FO supplementation and were included in the study. Median age was 68 (24-90) years, median weight was $70 \mathrm{~kg}(40-158)$ and $62 \%$ of 
participants were men. Patients received the standard drug treatment for their pathology.

The most frequent diagnosis was digestive neoplasm (42 \%) (Table 1) and the main indication for PN administration was paralytic ileus or intestinal failure. Overall, 40 patients stayed in ICU with a median LOS of 9.5 days (2-99). Among the studied patients, 8 died during the follow-up period (15.1\%).

The median length of treatment with $\mathrm{PN}$ was 19 (7-75) days. In Table 2, the amount of nutrients is described. Altogether, types of lipids administered to the patients were: $24 \%$ SO, $18.8 \%$ MCT, $36.6 \%$ olive oil, $20.6 \%$ FO. The most frequent emulsion used to supply lipids was a combination of Clinoleic ${ }^{\circ}$ with Omegaven .

Among the 23 septic patients, 22 (95.6\%) presented their highest values of GGT, AP or ALT during the septic event; while 23 (100\%) had their highest value of BIL simultaneously with the sepsis.

\section{Statistical results}

In the univariate model ANOVA, variation of LFTs was categorized and the number of patients classified in each group is shown in Table 3. ANOVA results showed that there were statistically significant differences regarding AP. Those patients with a higher decrease of AP had received higher doses of FO. The mean of the total dose received was $8.96 \mathrm{~g} / \mathrm{kg}$ in those with a high decrease of AP.

No significance was found between the total dose of FO and GGT; a trend towards significance was found in ALT and BIL (Table 3).

In the multivariate model, variation of LFTs was considered as a continuous variable and the following statistically significant results were obtained (Table 4 and Fig. 1):

- The variable associated with an increase in GGT values was sepsis, $B=5.03$ [CI $95 \%=0.29 / 9.76$ ], however, the total amount of FO administered was

Table 1 Diagnosis

\begin{tabular}{ll}
\hline Diagnostic & $\mathrm{n}(\%)$ \\
\hline Digestive neoplasm & $22(42 \%)$ \\
Pancreatitis & $7(13 \%)$ \\
Other digestive diseases & $6(11 \%)$ \\
Septic shock & $5(9 \%)$ \\
Multiple trauma & $4(8 \%)$ \\
Cardiovascular disease & $3(6 \%)$ \\
Other neoplasm & $3(6 \%)$ \\
Status epilepticus & $2(4 \%)$ \\
Respiratory disease & $1(2 \%)$ \\
Total & $53(100 \%)$ \\
\hline
\end{tabular}

associated with a decrease in GGT, B = -2.23 [CI $95 \%=-4.41 /-0.05]$.

- The increase of AP was associated with sepsis, B = 2.25 [CI $95 \%=0.43 / 4.07$ ] and a greater number of days in ICU, B $=0.08$ [CI $95 \%=0.03 / 0.14]$. On the other hand, the total amount of FO administered was associated with a decrease in AP, B $=-1.23$ [CI $95 \%=-2.07 /-0.37]$.

- ALT values only showed association with one variable: ALT decreased when the dose of FO administered increased $\mathrm{B}=-0.82$ [CI $95 \%=-1.19 /-0.44$ ].

- Finally, increases in BIL were only associated with a longer stay in ICU B = 1.97 [CI $95 \%=1.11 / 2.84$ ].

Determination coefficients $\left(\mathrm{R}^{2}\right)$ were consistent.

In the t-tests for safety variables, no differences were found in the serum TG levels: initial TG median value $1.7 \mathrm{mmol} / \mathrm{L}(1.1-3.6)$ and final TG median $1.7 \mathrm{mmol} / \mathrm{L}$ (1.0-3.4) $\quad(\mathrm{p}=0.666)$. With regards to coagulation markers, no differences, but a trend to significance, was found in PLC and INR values between the beginning and the end of treatment. PLC initial median $315 \times 10^{9}$ cells $/ \mathrm{L}\left(54 \times 10^{9}-1285 \times 10^{9}\right)$ vs. final median $368 \times 10^{9}$ cells $/ \mathrm{L}$ $\left(30 \times 10^{9}-834 \times 10^{9}\right)(\mathrm{p}=0.073)$; and INR initial median 1.22 $(0.94-2.56)$ vs. final median $1.2(0.78-1.194)$ ( $\mathrm{p}=0.059)$.

\section{Discussion}

This study demonstrated that, when administering FO supplementation to adult patients treated with PN during hospitalization, higher doses of FO were associated with an improvement in some liver parameters (GGT, $\mathrm{AP}$ and ALT), regardless of other factors that are normally associated with the alteration of these LFTs in patients undergoing PN treatment.

To our best knowledge, little research has been conducted on the management of liver alteration in adults during hospitalized PN treatment with FO. In fact, very few studies have focused on adults with SBS and PNALD [13, 15], while other aspects of the potential benefits of Omegaven ${ }^{\oplus}$ in reducing infections and improving postoperative health outcomes in the critically ill population have been well studied [16].

LFT alterations associated with $\mathrm{PN}$ is a frequent finding and can be related to many factors [17], among them intravenous lipid administration [18] since in 1982, Allardyce [19] reported on the prevention of cholestasis with the use of lipid reduction strategies. The cause appears to be at least partially associated with the composition of the SO lipid emulsions that aggravates or induces hepatocellular and systemic inflammatory effects [4]. Lipid metabolism results in lipid peroxidation and free radical formation, causing functional damage to hepatocytes, leading to reduced bile production and cholestasis in animal models. The higher oxidation susceptibility of $\omega-6$ 
Table 2 Main nutritional and analytical variables collected during the study

\begin{tabular}{lll}
\hline Variables & Median (range) & \\
\hline Body Mass Index (BMI) & $25.4(17.3-41.5)$ & \\
Parenteral Nutrition regimen & & \\
Total non-protein calorie (kcal/kg/d) & $16.7(7.7-28.3)$ & \\
Proteins (g/kg/day) & $1.04(0.43-1.88)$ & \\
Total fat (g/kg/day) & $0.61(0.22-1)$ & \\
Fish oil emulsion (g/kg/day) & $0.07(0.01-0.9)$ & \\
Dextrose (g/kg/day) & $2.73(1.17-4.58)$ & \\
Laboratory & Initial PN & Final PN \\
Serum albumin (g/L) & $28[15-42]$ & $30[15-38]$ \\
Serum prealbumin (mg/L) & $123(17.6-209)$ & $199(41.1-290)$ \\
C-Reactive protein (mg/L) & $186.4(3.6-405.6)$ & $133.7(2.4-339.5)$ \\
Bilirubin ( $\mu$ mol/L) & $8.25(2-151)$ & $7.0(2-358)$ \\
Alanine aminotransferase ( $\mu$ kat/L) & $0.32(0.11-4.67)$ & $0.55(0.11-5.83)$ \\
Alkaline phosphatase ( $\mu$ kat/L) & $1.4(0.5-11)$ & $3.0(0.6-14.20)$ \\
Gamma-glutamyl transferase & $1.29(0.17-8.51)$ & $4.28(0.16-46.72)$ \\
( $\mu$ kat/L) & & \\
Triglycerides (mmol/L) & $1.7(1.1-3.6)$ & $1.7(1.0-3.4)$ \\
Platelet count $\left(\times 10^{9}\right.$ cells/L) & $315(54-1285)$ & $368(30-834)$ \\
International normalized ratio (INR) & $1.22(0.94-2.56)$ & $1.20(0.78-1.94)$ \\
\hline
\end{tabular}

PUFAs requires antioxidant enrichment to counteract formation of reactive oxygen species, although currently utilized levels may not be sufficient [9]. Following these concerns, several alternatives were investigated and, although the precise mechanism by which these effects are triggered has yet to be clearly determined, parenteral FO administration shows several advantages that could explain the improvement in liver function. FO lipid emulsion lacks hepatotoxic phytosterols [20, 21]; improves biliary flow and cholestasis; and it has been shown to be effective in lowering triglyceride levels by increasing their clearance $[4,22]$. Owing to their high concentration of $\omega$ 3 PUFAs [20], FO has an anti-inflammatory potential via competitive inhibition of the $\omega-6$ PUFAs-derived proinflammatory eicosanoids [23] linked to liver injury causing both apoptosis and necrosis in many types of liver disease (cholestasis, non-alcoholic fatty liver disease, alcoholic cirrhosis and hepatitis) [5]. That is why some authors propose, in line with our results, that increasing the hepatic content of $\omega-3$ PUFAs could decrease inflammatory activity in acute hepatitis with alleviation of hepatic injury and inflammation [23]. Parenteral FO is enriched with high levels of the antioxidant $\alpha$-tocopherol to counteract possible oxidative risk [9], while other lipids contain $\gamma$ tocopherol and have less anti-oxidative effects [24].

Table 3 Analyses of variance between doses of fish oil and the variation of each liver parameter

\begin{tabular}{|c|c|c|c|c|c|c|}
\hline \multicolumn{7}{|c|}{ Gamma-glutamyl transferase variation } \\
\hline Variation category & High increase & Moderate increase & No modification & Moderate decrease & High decrease & $p$ \\
\hline$n$ & 10 & 8 & 9 & 19 & 7 & 0.156 \\
\hline FO dose mean ${ }^{a}$ & 1.69 & 0.64 & 1.08 & 3.61 & 4.47 & \\
\hline Rank & $0.01-6.44$ & $0.02-2.79$ & $0.04-2.25$ & $0.17-22.56$ & $0.4-13.12$ & \\
\hline \multicolumn{7}{|c|}{ Alkaline phosphatase variation } \\
\hline Variation category & High increase & Moderate increase & No modification & Moderate decrease & High decrease & $p$ \\
\hline $\mathrm{n}$ & 8 & 16 & 16 & 7 & 6 & 0.000 \\
\hline FO dose mean ${ }^{a}$ & 2.22 & 1.16 & 1.72 & 1.41 & 8.96 & \\
\hline Rank & $0.01-6.44$ & $0.02-2.79$ & $0.05-12.6$ & $0.3-3.6$ & $3.36-22.56$ & \\
\hline \multicolumn{7}{|c|}{ Alanine aminotransferase variation } \\
\hline Variation category & High increase & Moderate increase & No modification & Moderate decrease & High decrease & $p$ \\
\hline$n$ & 7 & 10 & 23 & 7 & 6 & 0.094 \\
\hline FO dose mean ${ }^{a}$ & 0.66 & 0.57 & 2.73 & 3.77 & 5.32 & \\
\hline Rank & $0.01-1.52$ & $0.02-2.47$ & $0.04-13.12$ & $0.24-12.6$ & $0.3-22.56$ & \\
\hline \multicolumn{7}{|l|}{ Bilirubin variation } \\
\hline Variation category & High increase & Moderate increase & No modification & Moderate decrease & High decrease & $p$ \\
\hline$n$ & 5 & 11 & 15 & 14 & 8 & 0.057 \\
\hline FO dose mean ${ }^{a}$ & 1.94 & 0.71 & 1.35 & 3.69 & 5.28 & \\
\hline Rank & $0.3-6.44$ & $0.02-1.63$ & $0.02-3.6$ & $0.22-13.12$ & $0.03-22.56$ & \\
\hline
\end{tabular}

FO fish oil

SD Standard deviation

${ }^{\mathrm{a}}$ Mean of the total dose of fish oil emulsion administered, expressed in $\mathrm{g} / \mathrm{kg}$

Figures marked in bold are statistically significant in the study 
Table 4 Multiple lineal regressions between liver parameters variation and four factors that can affect them

\begin{tabular}{|c|c|c|c|c|c|c|c|c|}
\hline \multicolumn{9}{|l|}{ Liver parameter } \\
\hline \multirow[t]{2}{*}{ Variable } & \multicolumn{2}{|c|}{ Gamma-glutamyl transferase } & \multicolumn{2}{|l|}{ Alkaline phosphatase } & \multicolumn{2}{|c|}{ Alanine aminotransferase } & \multicolumn{2}{|l|}{ Bilirubin } \\
\hline & B Cl $95 \%$ & $p$ & B Cl $95 \%$ & $p$ & B Cl $95 \%$ & $\mathrm{p}$ & B Cl $95 \%$ & $p$ \\
\hline Sepsis & $5.03[0.29 / 9.76]$ & 0.038 & $2.25[0.43 / 4.07]$ & 0.017 & $0.36[-0.46 / 1.18]$ & 0.38 & $-11.04[-39.2 / 17.1]$ & 0.43 \\
\hline Total vegetable lipids dose & $-0.077[-0.31 / 0.152]$ & 0.50 & $-0.03[-1.11 / 0.06]$ & 0.175 & $-0.11[-0.05 / 0.03]$ & 0.56 & $-0.47[-1.82 / 0.88]$ & 0.49 \\
\hline Total fish oil dose & $-2.23[-4.41 /-0.05]$ & 0.045 & $-1.23[-2.07 /-0.37]$ & 0.005 & $-0.82[-1.19 /-0.44]$ & 0.000 & $-1.81[-14.8 / 11.1]$ & 0.78 \\
\hline $\begin{array}{l}\text { Length of stay in the intensive } \\
\text { care unit }\end{array}$ & $0.05[-0.10 / 0.20]$ & 0.50 & $0.08[0.03 / 0.14]$ & 0.005 & $-0.01[-0.04 / 0.01]$ & 0.31 & $1.97[1.11 / 2.84]$ & 0.000 \\
\hline Determination Coefficient & $R^{2}: 0.15$ & & $R^{2}: 0.33$ & & $R^{2}: 0.31$ & & $R^{2}: 0.34$ & \\
\hline
\end{tabular}

Cl confidence interval

Figures marked in bold are statistically significant in the study

Finally, FO-containing emulsions improve splanchnic blood flow $[2,25,26]$.

Clinically, the relationship between $\omega-3$ FAs and liver function has been more intensively studied in children with SBS and PNALD. Since Gura et al. [27, 28] published their first papers on the subject, many publications have appeared showing different strategies to deal with this problem [29-32]. Currently, even if there is some concern that the improvement found in PNALD in children might be due to lipid dose reduction rather than the type of lipid administered [33] a systematic review by Seida et al. [34] concluded that the best available evidence at present supports the use of $\omega$-3 FAs supplementation to improve biochemical outcomes of PNALD in young children dependent on PN.

There are some studies on FO administration in adult patients with SBS and PNALD. Xu et al. [13] in a 15patient study, found that bilirubin normalized after 4 weeks of treatment when SO content of PN was partially replaced by FO up to $10 \mathrm{~g}$ daily, about $0.15-0.2 \mathrm{~g} /$ $\mathrm{kg} / \mathrm{d}$. Differences in ALT values were also statistically significant. Nevertheless, even when GGT values also decreased, statistically significant differences were not found. Venecourt et al. [35] showed that after changing one patient to a total FO emulsion, bilirubin decreased over the ensuing 8 weeks, with normalization of transaminases in 10 weeks. In the case of Burns et al. [36], FO administration decreased total bilirubin; AP initially increased but subsequently declined to a stable pretreatment value and transaminases slightly decreased initially and then remained stable. In a patient described by Jurewitsch et al. [37] the change to a blend of FO plus SO curtailed the increase in liver enzymes but did not reduce total bilirubin levels. It was subsequently decided to give only FO and total bilirubin levels fell rapidly. The main difference between those patients and ours is that all those patients had established liver disease when they started treatment with FO and they had developed liver disease after a long period of PN treatment: 110 days in the Jurewitsch study [37], 3 years in the Venecourt paper [35] and
5 years in Burns [36]. In the Xu study [13], patients developed PNALD after 2-19 months of treatment with PN. In our patients, we found a significant decrease in AP, GGT and ALT values, even when they had received PN for a short period of time (median of 19 days) and they did not achieve this status of an advanced form of liver disease. On the other hand, the main commercial emulsions that we used to provide lipids were the combination of Clinoleic ${ }^{\circ}$ with Omegaven ${ }^{\circ}$ and no studies yet show whether the immunoneutral effect of high amounts of olive oil could contribute to the improvement of some liver parameters.

Studies on LFT alterations and hospitalization-PN administration using FO in adults are scarce. Our results on ALT improvement with FO agree with those found in the bibliography. In patients undergoing liver transplantation, Zhu et al. [38] found that ALT improved significantly after 9 days of FO supplementation $(0.2 \mathrm{~g} / \mathrm{kg} / \mathrm{d})$ compared with traditional PN support (LCT:MCT). Total lipid intake was $1 \mathrm{~g} / \mathrm{kg} / \mathrm{d}$. In a randomized, double-blind trial comparing SMOFlipid $^{\bullet}$ with olive oil in patients following major abdominal surgery or large cranio-maxillo-facial resections for cancer, Piper et al. [2] found that significantly lower ALT values were observed at days 2 and 5 in the SMOFlipid $^{\circ}$ group. Pawlik et al. [39] randomized a group of lowweight newborn infants to receive an intravenous mixture of Clinoleic ${ }^{\circledR}$ plus Omegaven ${ }^{\circledR}$ or just a Clinoleic ${ }^{\circledR}$ parenteral emulsion. They found that, after 22 days of PN administration, 3 infants in the FO group developed cholestasis compared with 20 infants in the standard group (RR 0.18; $95 \% \mathrm{CI}, 0.055-0.56$ ). The results of these publications agree with our results in the sense that they treat patients without PNALD, during a similar period of time and they have similar improvement in the LFTs. Our study goes beyond the previous ones by finding that increasing the dose of FO increases the improvement of ALT values independently of other factors.

Hepatic lipid accumulation is the result of an imbalance in de novo lipogenesis, FA oxidation, and/or TG secretion rates [40] and these mechanisms may also be involved in hepatic steatosis (with ALT and GGT elevation) occurring 

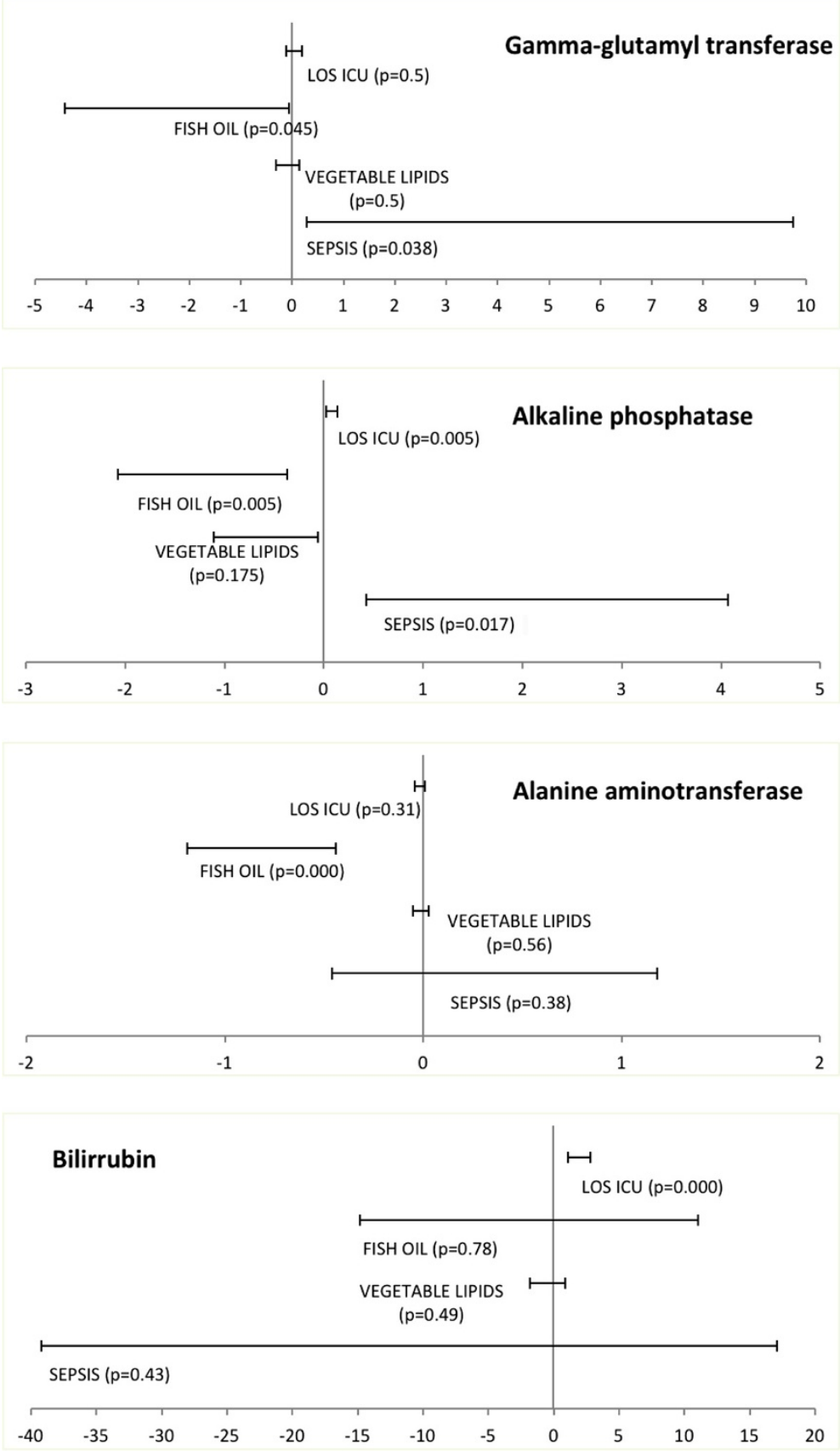

LOS ICU: Length of stay in the intensive care unit

Fig. 1 Representation of the B $95 \%$ confidence interval of the four risk factors associated to each liver function test

as a consequence of PN [17]. Increased intake of $\omega-3$ PUFAs has been found to protect against fatty liver disease since it reduces hepatic lipogenesis and decreases the level of inflammation. Benefits have been noticed in patients with non-alcoholic fatty liver disease [41] and in animal studies on PNALD [21, 22, 40].

Among chronic liver diseases, the association between adipokines and non alcoholic fatty liver disease is well established. Adiponectine, the most abundant adiposespecific adipokine, decreases hepatic and systematic insulin resistance, and attenuates liver inflammation and fibrosis. That is why recent therapeutic strategies have focused on the indirect upregulation of adiponectin. In addition to its relation with chronic liver alteration, early data suggest that plasma levels of adiponectin are decreased in critical illness. Nevertheless, the detailed 
mechanisms underlying its hepato-protective functions remain largely uncharacterized [42-44].

No safety problems were detected in our patients since no significant differences were found when comparing serum levels of TG and coagulation parameters. The product labeling for the FO-based ILE indicates that it could increase bleeding risk, nevertheless authors such as Bays [45] and Harris [46] concluded that $\omega-3$ PUFAs do not increase this risk. Results from Puder et al. [47] are in line with ours suggesting that FO has a limited effect on coagulation since the infants receiving FO had lower INR values and increased platelet counts compared with those treated with SO, moreover they point out that this increment in platelet counts suggests a possible improvement in liver function. The same authors [44] reported that the rate of hypertriglyceridemia in children receiving $\mathrm{FO}$ was lower than in children receiving SO; and TG levels in the FO group were lower $(\mathrm{P}=0.0002)$ and declined faster than in the SO group. Differences in levels and trends were statistically significant $(\mathrm{P}<0.0001)$.

Some limitations can be attributed to this present study, such as the retrospective collection of data, even when the high degree of computerization reached in our hospital has allowed us to find almost every single piece of data that we wanted to study. Another possible limitation is that we did not study the amount of $\alpha$-tocopherol administered. This could be important, and would be an interesting further avenue to explore since it is presently unclear whether the beneficial effects are caused by FO, $\alpha$-tocopherol or by the exact mixture of the lipid components. On the other hand, histopathological measurements could have been undertaken to prove subclinical damage but, since they are not standard clinical procedures, they were not performed due to both ethical and financial concerns.

\section{Conclusions}

Although the use of FO lipid emulsion as monotherapy is still questioned, some groups have shown that children receiving PN with Omegaven monotherapy improve their LFTs and do not develop any signs of essential fatty acid deficiency or growth retardation [22]. Studying the effect of FO in adults, we have seen a clear improvement in some liver parameters (ALT and GGT) in a group of adult patients treated with $\mathrm{PN}$ during hospitalization. In addition, this improvement is larger when the dose of FO is increased. Since FO has been tried as a single lipid administered as $\mathrm{PN}$ in children and taking our results into account, it would be of interest to use higher doses of FO in those adult patients who develop liver parameter alterations during hospitalization with PN treatment. Randomized trials are needed to confirm this proposition.

\section{Abbreviations}

ALT: Alanine aminotransferase; AP: Alkaline phosphatase; BIL: Bilirubin;

FAs: Fatty acids; FO: Fish oil; GGT: Gamma-glutamyl transferase; ICU: Intensive care unit; LFTs: Liver function tests; LOS: Length of stay; PN: Parenteral nutrition; INR: International normalized ratio; PNALD: Parenteral nutrition associated liver disease; PLC: Platelets count; PUFAs: Polyunsaturated fatty acids; TG: Triglycerides.

\section{Competing interests}

The authors declare that they have no competing interests.

This research received no specific grant from any funding agency, commercial or not-for-profit sectors.

\section{Authors' contributions}

MBT designed the study, carried it out, analysed the data and wrote the manuscript, ELB carried out the study and corrected the manuscript, RJM corrected the manuscript; JRT revised the manuscript; and JLT designed the study, did the statistical analysis and corrected the manuscript. All authors read and approved the final manuscript.

\section{Acknowledgements}

We thank Maren White for editorial support in the preparation of this manuscript.

Part of this work was presented in poster form at the European Society for Parenteral and Enteral Nutrition Annual Congress; September 8-11, 2012; Barcelona, Spain.

\section{Author details}

${ }^{1}$ Pharmacy Department, Hospital Universitari Bellvitge. IDIBELL. C/Feixa Llarga s/n. 08907 L'Hospitalet de Llobregat, Barcelona, Spain. ${ }^{2}$ Preventive Medicine Department, Hospital Universitari Bellvitge. IDIBELL. C/Feixa Llarga s/n. 08907 L'Hospitalet de Llobregat, Barcelona, Spain.

Received: 10 February 2015 Accepted: 9 June 2015

Published online: 02 July 2015

\section{References}

1. El-Badry AM, Graf R, Clavien PA. Omega 3 - Omega 6: What is right for the liver? J Hepatol. 2007;47:718-25.

2. Piper SN, Schade I, Beschmann RB, Maleck WH, Boldt J, Röhm KD. Hepatocellular integrity after parenteral nutrition: comparison of a fish-oilcontaining lipid emulsion with an olive-soybean oil-based lipid emulsion. Eur J Anaesthesiol. 2009;26:1076-82.

3. Nanji AA, Anderson FH. Sensitivity and specificity of liver function tests in the detection of parenteral nutrition-associated cholestasis. JPEN J Parenter Enteral Nutr. 1985;9:307-8.

4. Visschers RGJ, Olde Damink SWM, Gehlen JMLG, Winkens B, Soeters PB, van Gemert WG. Treatment of hypertriglyceridemia in patients receiving parenteral nutrition. JPEN J Parenter Enteral Nutr. 2011;35:610-5.

5. Tillman EM. Review and clinical update on parenteral nutrition-associated liver disease. Nutr Clin Pract. 2013;28:30-9.

6. González Santos A, Tamez Pérez HE, Gutiérrez Hermosillo H, de León D, González E. Significant liver function tests abnormalities related to parenteral nutrition and its association with mortality. Nutr Hosp. 2011;26:243.

7. Diaz de Leon González E, Gutiérrez Hermosillo H, Piquet Uscanga YO, García Vallejo C, Vázquez Martínez CA, Alvarado Zaldivar G, et al. Risk factors for abnormal liver function tests of parenteral nutrition in a referral hospital in Mexico. Nutr Hosp. 2011;26:729-36.

8. Carter BA, Shulman RJ. Mechanisms of disease: update on the molecular etiology and fundamentals of parenteral nutrition associated cholestasis. Nat Clin Pract Gastroenterol Hepatol. 2007;4:277-87.

9. De Meijer VE, Gura KM, Meisel JA, Le HD, Puder M. Parenteral fish oil monotherapy in the management of patients with parenteral nutritionassociated liver disease. Arch Surg. 2010;145:547-51.

10. Llop JM, Virgili N, Moreno-Villares JM, García-Peris P, Serrano T, Forga M, et al. Phytosterolemia in parenteral nutrition patients: implications for liver disease development. Nutrition. 2008:24:1145-52.

11. Carter BA, Taylor OA, Prendergast DR, Zimmerman TL, Von Furstenberg R, Moore DD, et al. Stigmasterol, a soy lipid-derived phytosterol, is an antagonist of the bile acid nuclear receptor FXR. Pediatr Res. 2007;62:301-6.

12. Saayman BD. The use of alternative emulsions in paediatric and neonatal parenteral nutrition. S Afr J Clin Nutri. 2011;24:S32-34. 
13. Xu Z, Li Y, Wang J, Wu B, Li J. Effect of omega-3 polyunsaturated fatty acids to reverse biopsy-proven parenteral nutrition-associated liver disease in adults. Clin Nutr. 2012;31:217-23.

14. Grau T, Bonet A, Rubio M, Mateo D, Farré M, Acosta JA, Blesa A, Montejo JC, de Lorenzo AG, Mesejo A; Working Group on Nutrition and Metabolism of the Spanish Society of Critical Care. Liver dysfunction associated with artificial nutrition in critically ill patients. Crit Care. 2007;11:R10.

15. Vanek WW, Seidner DL, Allen P, Bistrian B, Collier S, Gura K, et al. A.S.P.E.N. position paper: Clinical role for alternative intravenous fat emulsions. Nutr Clin Pract. 2012;27:150-92.

16. Heller AR, Rössler S, Litz RJ, Stehr SN, Heller SC, Koch R, et al. Omega-3 fatty acids improve the diagnosis-related clinical outcome. Crit Care Med. 2006;34:972-9.

17. Kopec KL, Burns D. Nonalcoholic fatty liver disease: a review of the spectrum of disease, diagnosis, and therapy. Nutr Clin Pract. 2011;26:565-76.

18. Badia-Tahull MB, Leiva-Badosa E, Llop-Talaverón J, Figueras-Suriol A, Quirante-Cremades A, Tubau-Molas M, et al. Liver function test alterations associated with parenteral nutrition in hospitalized adult patients: incidence and risk factors. Nutr Hosp. 2012;27:1279-85.

19. Allardyce DB. Cholestasis caused by lipid emulsions. Surg Gynecol Obstet. 1982;154:641-7.

20. Nehra D, Fallon EM, Carlson SJ, Potemkin AK, Hevelone ND, Mitchell PD, et al. Provision of a soy-based intravenous lipid emulsion at $1 \mathrm{~g} / \mathrm{kg} / \mathrm{d}$ does not prevent cholestasis in neonates. JPEN J Parenter Enteral Nutr. 2013;37:498-505.

21. Prince $E$, Lazare FB, Treem WR, Xu J, lqbal J, Pan X, et al. $\omega-3$ fatty acids prevent hepatic steatosis, independent of PPAR-a activity, in a murine model of parenteral nutrition-associated liver disease. JPEN J Parenter Enteral Nutr. 2013;38:608-16.

22. Fallon EM, Le HD, Puder M. Prevention of parenteral nutrition-associated liver disease: role of omega-3 fish oil. Curr Opin Organ Transplant. 2010;15:334-40.

23. Schmöcker C, Weylandt KH, Kahlke L, Wang J, Lobeck H, Tiegs G, et al. Omega-3 fatty acids alleviate chemically induced acute hepatitis by suppression of cytokines. Hepatology. 2007;45:864-9.

24. Goulet $\mathrm{O}$, Joly F, Corriol O, Colomb-Jung V. Some new insights in intestina failure-associated liver disease. Curr Opin Organ Transplant. 2009;14:256-61.

25. Pscheidl E, Schywalsky M, Tschaikowsky K, Böke-Pröis T. Fish oilsupplemented parenteral diets normalize splanchnic blood flow and improve killing of translocated bacteria in a low-dose endotoxin rat model. Crit Care Med. 2000;28:1489-96.

26. Kurihara T, Adachi Y, Yamagata M, Abe K, Akimoto M, Hashimoto $H$, et al Role of eicosapentaenoic acid in lipid metabolism in the liver, with special reference to experimental fatty liver. Clin Ther. 1994;16:830-7.

27. Gura KM, Parsons SK, Bechard LJ, Henderson T, Dorsey M, Phipatanakui W, et al. Use of a fish oil-based lipid emulsion to treat essential fatty acid deficiency in a soy allergic patient receiving parenteral nutrition. Clin Nutr. 2005;24:839-47.

28. Gura KM, Duggan CP, Collier SB, Jennings RW, Folkman J, Bistrian BR, et al. Reversal of parenteral nutrition-associated liver disease in two infants with short bowel syndrome using parenteral fish oil: implications for future management. Pediatrics. 2006;118:e197-201.

29. Angsten G, Finkel Y, Lucas S, Kassa AM, Paulsson M, Lilja HE. Improved outcome in neonatal short bowel syndrome using parenteral fish oil in combination with $\omega-6 / 9$ lipid emulsions. JPEN J Parenter Enteral Nutr. 2012;36:587-95.

30. Soden JS, Lovell MA, Brown K, Partrick DA, Sokol RJ. Failure of resolution of portal fibrosis during omega-3 fatty acid lipid emulsion therapy in two patients with irreversible intestinal failure. J Pediatr. 2010;156:327-31.

31. Yang CF, Lee M, Valim C, Hull MA, Zhou J, Jones BA, et al. Persistent alanine aminotransferase elevations in children with parenteral nutrition-associated liver disease. J Pediatr Surg. 2009:44:1084-7. discussion 1087-88.

32. Park KT, Nespor C, Kerner Jr J. The use of Omegaven in treating parenteral nutrition-associated liver disease. J Perinatol. 2011;31 Suppl 1:S57-60.

33. Skouroliakou M, Konstantinou D, Agakidis C, Delikou N, Koutri K, Antoniadi $\mathrm{M}$, et al. Cholestasis, bronchopulmonary dysplasia, and lipid profile in preterm infants receiving MCT/ $\omega$-3-PUFA-containing or soybean-based lipid emulsions. Nutr Clin Pract. 2012;27:817-24.

34. Seida JC, Mager DR, Hartling L, Vandermeer B, Turner JM. Parenteral $\omega-3$ fatty acid lipid emulsions for children with intestinal failure and other conditions: a systematic review. JPEN J Parenter Enteral Nutr. 2013;37:44-55.
35. Venecourt-Jackson E, Hill SJ, Walmsley RS. Successful treatment of parenteral nutrition-associated liver disease in an adult by use of a fish oil-based lipid source. Nutrition. 2013;29:356-8.

36. Burns DL, Gill BM. Reversal of parenteral nutrition-associated liver disease with a fish oil-based lipid emulsion (Omegaven) in an adult dependent on home parenteral nutrition. JPEN J Parenter Enteral Nutr. 2013;37:274-80.

37. Jurewitsch B, Gardiner G, Naccarato M, Jeejeebhoy KN. Omega-3-enriched lipid emulsion for liver salvage in parenteral nutrition-induced cholestasis in the adult patient. JPEN J Parenter Enteral Nutr. 2011;35:386-90.

38. Zhu X, Wu Y, Qiu Y, Jiang C, Ding Y. Effect of parenteral fish oil lipid emulsion in parenteral nutrition supplementation combined with enteral nutrition support in patients undergoing pancreaticoduodenectomy. JPEN J Parenter Enteral Nutr. 2013;37:236-42.

39. Pawlik D, Lauterbach R, Walczak M, Hurkata J, Sherman MP. 2013. Fish-oil fat emulsion supplementation reduces the risk of retinopathy in very low birth weight infants: a prospective, randomized study. JPEN J Parenter Enteral Nutr. 2014;38:711-6.

40. Musso G, Gambino R, Cassader M. Recent insights into hepatic lipid metabolism in non-alcoholic fatty liver disease (NAFLD). Prog Lipid Res. 2009;48:1-26.

41. Oya J, Nakagami T, Sasaki S, Jimba S, Murakami K, Kasahara T, et al. Intake of n-3 polyunsaturated fatty acids and non-alcoholic fatty liver disease: a crosssectional study in Japanese men and women. Eur J Clin Nutr. 2010;64:1179-85.

42. Di Minno MN, Russolillo A, Lupoli R, Ambrosino P, Di Minno A, Tarantino G. Omega-3 fatty acids for the treatment of non-alcoholic fatty liver disease. World J Gastroenterol. 2012;18:5839-47.

43. Finelli C, Tarantino G. What is the role of adiponectin in obesity related non-alcoholic fatty liver disease? World J Gastroenterol. 2013;19:802.

44. Yamamotoa T, Kajikawab Y, Otania S, Yamadaa Y, Takemotob S, Hirotab M, et al. Protective effect of eicosapentaenoic acid on insulin resistance in hyperlipidemic patients and on the postoperative course of cardiac surgery patients: the possible involvement of adiponectin. Acta Med Okayama. 2014;68:349-61.

45. Bays HE. Safety considerations with omega-3 fatty acid therapy. Am J Cardiol. 2007:99:35C-43C.

46. Harris WS. Expert opinion: omega-3 fatty acids and bleeding-cause for concern? Am J Cardiol. 2007:99:44C-6C

47. Puder M, Valim C, Meisel JA, Le HD, de Meijer VE, Robinson EM, et al. Parenteral fish oil improves outcomes in patients with parenteral nutritionassociated liver injury. Ann Surg. 2009;250:395-402.

\section{Submit your next manuscript to BioMed Central and take full advantage of:}

- Convenient online submission

- Thorough peer review

- No space constraints or color figure charges

- Immediate publication on acceptance

- Inclusion in PubMed, CAS, Scopus and Google Scholar

- Research which is freely available for redistribution 